in vivo $35: 385-391(2021)$

doi:10.21873/invivo.12269

\title{
Adolescents and Young Adults (AYAs) With Initially Localized and Metastatic Bone Sarcomas: A Retrospective Single Center Analysis of Side Effect Management
}

\author{
CHRISTOPH MINICHSDORFER ${ }^{1}$, OSKAR STEINBRECHER ${ }^{1}$, MARITA KÖLZ ${ }^{2}$, \\ MAXIMILIAN SCHMID ${ }^{3}$, MARKUS RADERER ${ }^{1}$, THOMAS BRODOWICZ ${ }^{1}$ and WOLFGANG LAMM ${ }^{1}$ \\ ${ }^{1}$ Clinical Division of Oncology, Department of Medicine I, \\ Medical University of Vienna - General Hospital, Vienna, Austria; \\ ${ }^{2}$ Department of Pathology, Medical University of Vienna - General Hospital, Vienna, Austria; \\ ${ }^{3}$ Department of Radiation Oncology, Medical University of Vienna - General Hospital, Vienna, Austria
}

\begin{abstract}
Background/Aim: Ewing sarcoma (ES) and osteosarcomas (OSA) are the most common bone tumor types in adolescents and young adults (AYA). Therapy management of these tumors consists of preoperative chemotherapy, operation, and postoperative chemotherapy. The aim of this study was to evaluate the efficacy and tolerability of EURAMOS-A and EURO E.W.I.N.G. protocols. Patients and Methods: We retrospectively evaluated 31 patients between 18 and 39 years of age with ES and OSA treated at the Department of Medicine I, Clinical Division of Oncology. Patients with ES were treated according the EURO E.W.I.N.G protocol, whereas patients with OSA according to the EURAMOS-1 protocol. Results: Most frequent tumor sites for ES were thorax and pelvis, each $33 \%$. Eight patients had initially localized disease (67\%). A median of 3 cycles of full dose chemotherapy could be administered. Nine patients had a dose reduction (75\%). Most common reason for dose reduction was prolonged aplasia (67\%). Overall response rate (ORR) was $33 \%$. For OSA patients, the most frequent tumor site was the lower extremity (58\%). Sixteen patients (84\%) had initially localized disease. A median of only 9 cycles of EURAMOS-1 in full dose could be administered. Most common reason for dose reduction was elevated
\end{abstract}

This article is freely accessible online.

All Authors contributed equally to this work.

Correspondence to: Wolfgang Lamm, MD, Medical University of Vienna, Department of Internal Medicine I, Oncology, Währingergürtel 18-20, 1090 Vienna, Austria. Tel: +43 014040044450 Fax: +43 014040044570, e-mail: wolfgang.lamm@meduniwien.ac.at

Key Words: Adolescents and young adults, bone sarcomas, Ewing sarcomas, osteosarcomas, EURAMOS-A, EURO E.W.I.N.G. methotrexate level (53\%) and ORR was 90\%. Conclusion: The two studied protocols were well-tolerated in the AYA patients included in this study. Dose reductions instead of dose delays should be considered when side effects occur.

Primary malignant tumors of osseous origin are rare and include tumors of the Ewing family, as well as osteosarcomas. Ewing family tumors (EFTs) comprise Ewing sarcomas (ES), extraosseous ES, Askin tumors, chondrosarcomas and primitive neuroectodermal tumors (PNET), and rank among the most common malignant tumors of the bone in children and adults (1). About one quarter of all EFTs are diagnosed with overt metastatic disease and metastases predominantly occur in the lungs, followed by bone and bone marrow (2). However, without systemic therapy, even patients with initially localized disease will develop systemic relapses in the vast majority, suggesting that almost all patients have upfront microscopic disease in distant organs (3).

Over the last decades, improvements in the outcome of patients with EFT have been achieved by multimodal therapeutic concepts such as local therapy (surgery and/or radiotherapy), and aggressive multi-agent chemotherapy, including alkylating agents, vincristine, and anthracyclines with or without etoposide (4). In Europe, the most commonly applied contemporary multiagent chemotherapy regimen in EFT patients is the EURO E.W.I.N.G. protocol consisting of 4 drugs (vincristine, ifosfamide, doxorubicin, and etoposide; VIDE) administered every 3 weeks. This regimen is followed by local therapy and thereafter, a chemotherapy combination of vincristine, adriablastine, and ifosfamide (VAI) (male) or vincristine, adriblastine, and cyclophosphamide (VAC) (female) every 3 weeks is given (5).

Osteosarcoma (OSA) is a primary malignant bone tumor, with an incidence of 3 cases per million people per year (6). Furthermore, OS is the most common cancer in adolescence 
Table I. Baseline characteristics for patients with osteosarcoma $(n=19)$.

\begin{tabular}{|c|c|c|c|}
\hline Characteristic & $\mathrm{n}(\%)$ & Characteristic & $\mathrm{n}(\%)$ \\
\hline Gender & & Dose reduction & \\
\hline Male & $11(58 \%)$ & $75 \%$ & $13(68 \%)$ \\
\hline Female & $8(42 \%)$ & $50 \%$ & $6(32 \%)$ \\
\hline Age* (years) & $27(18-39)$ & Reason for dosereduction & \\
\hline Tumor site & & Missing MTX degradation & $10(53 \%)$ \\
\hline Upper extremity & $1(5 \%)$ & Prolonged aplasia & $8(42 \%)$ \\
\hline Lower extremitiy & $11(58 \%)$ & PS greater 1 & $1(5 \%)$ \\
\hline Chest & $1(5 \%)$ & Response to chemotherapy & \\
\hline Pelvis/abdomen & $3(16 \%)$ & PR & $1(5 \%)$ \\
\hline Face/neck & $3(16 \%)$ & PD & $2(10 \%)$ \\
\hline Extend of disease at diagnosis & & $\operatorname{NED}(n=16)$ & $16(85 \%)$ \\
\hline Primary localized & $16(84 \%)$ & Radiation therapy & \\
\hline Primary metastatic & $3(16 \%)$ & Yes & $1(5 \%)$ \\
\hline Metastatic site & & No & $18(95 \%)$ \\
\hline Lung & $3(100 \%)$ & Surgery after neoadjuvant EURAMOS & \\
\hline Liver & $2(67 \%)$ & Yes & $13(68 \%)$ \\
\hline Other & $3(100 \%)$ & No & $6(32 \%)$ \\
\hline Chemotherapy & & Response criteria after surgery $(n=13)$ & \\
\hline EURAMOS & $19(100 \%)$ & Responder & $1(8 \%)$ \\
\hline Cycles* & $19(5-21)$ & Non-responder & $12(92 \%)$ \\
\hline Cycles full dosage* & $9(1-14)$ & Secondline chemotherapy & \\
\hline Cycles full dose & & Pembrolizumab & $3(16 \%)$ \\
\hline 1 cycle & $1(5 \%)$ & Ifosfamide & $5(26 \%)$ \\
\hline 2 cycles & $1(5 \%)$ & Other & $2(10 \%)$ \\
\hline 3 cycles & $1(5 \%)$ & No second line chemotherapy & $9(47 \%)$ \\
\hline 5 cycles & $1(5 \%)$ & Response to second line chemotherapy & \\
\hline 6 cycles & $2(10 \%)$ & PR & $3(30 \%)$ \\
\hline 7 cycles & $3(15 \%)$ & SD & $5(50 \%)$ \\
\hline 9 cycles & $2(10 \%)$ & $\mathrm{PD}$ & $2(20 \%)$ \\
\hline 10 cylces & $1(5 \%)$ & Current status & \\
\hline 11 cycles & $4(21 \%)$ & Dead & $5(26 \%)$ \\
\hline 13 cycles & $2(10 \%)$ & Alive & $14(74 \%)$ \\
\hline \multirow[t]{2}{*}{14 cycles } & $1(5 \%)$ & Progression-free survival* (months) & $36.3(11.7-60.9)$ \\
\hline & & Overall survival & n.r. \\
\hline
\end{tabular}

*Data presented as median (range). MTX, Methotrexate; PS, performance status; PR, partial remission; PD, progressive disease; NED, no evidence of disease; SD, stable disease; n.r., not reached.

with a second peak incidence in the second decade of life (7, 8). Treatment of osteosarcomas with multimodal therapies results in a 3 year event free survival of approximately 60$70 \%$ (9). The most commonly used multiagent chemotherapy protocol is the EURAMOS-1 protocol. According to EURAMOS-1, cisplatin, doxorubicin and high-dose methotrexate (MAP) is given in the preoperative as well in the postoperative setting (10). The most commonly used second-line chemotherapy in patients with OSA is ifosfamide with or without etoposide. $(11,12)$ Combination therapy results in an overall response rate of approximately $59 \%$ in newly diagnosed metastatic OSA patients (12).

As our institution is a tertiary referral center for sarcoma patients, and both OSA and ES occur in adolescents and young adults, the objective of this monocentric retrospective analysis was to investigate the efficacy and tolerability of the two protocols (EURO E.W.I.N.G. and EURAMOS) in adolescents and young adults with ES and OSA.

\section{Patients and Methods}

Patients. This single-center retrospective analysis was performed on a cohort of 31 consecutive patients with histologically proven ES or OSA (either localized or metastatic), including OSA and ES who were treated at the Medical University of Vienna, Department of Oncology between 2013 and 2019. Histology examinations were sent to our referral pathology centre for re-evaluation and confirmation of diagnosis at our pathology department. Baseline characteristics are outlined in Tables I and II. Primary tumor assessment was done using magnetic resonance imaging (MRI) and/or computed tomography (CT), with additional CT scans of the chest and the abdomen to screen for metastases. After finalization of postoperative systemic treatment, follow-up using CT-scans and MRI, if indicated, was 
Table II. Baseline characteristics for patients with Ewing sarcomas $(n=12)$.

\begin{tabular}{|c|c|c|c|}
\hline Characteristic & $\mathrm{n}(\%)$ & Characteristic & $\mathrm{n}(\%)$ \\
\hline Gender & & Dose reduction $(\mathrm{n}=9)$ & $75 \%$ \\
\hline Male & $10(83 \%)$ & $75 \%$ & $7(78 \%)$ \\
\hline Female & $2(17 \%)$ & $50 \%$ & $2(22 \%)$ \\
\hline Age* (years) $^{*}$ & $24(18-37)$ & Reason for dose reduction $(n=9)$ & \\
\hline Tumor site & & Prolonged aplasia & $6(67 \%)$ \\
\hline Lower extremity & $2(17 \%)$ & Neurotoxicity due to Ifosfamide & $3(33 \%)$ \\
\hline Thorax & $4(33 \%)$ & Methylene blue (n=3) & $3(100 \%)$ \\
\hline Pelvis & $4(33 \%)$ & Response to chemotherapy & \\
\hline Abdomen & $2(17 \%)$ & $\mathrm{CR}$ & $1(8 \%)$ \\
\hline Primary disease & & PR & $3(25 \%)$ \\
\hline Initially localized & $8(67 \%)$ & PD & $1(8 \%)$ \\
\hline Initially metastatic & $4(33 \%)$ & NED & $7(58 \%)$ \\
\hline Metastatic site & & Radiation therapy & \\
\hline Lung & $4(100 \%)$ & Yes & $6(50 \%)$ \\
\hline Liver & $2(50 \%)$ & No & $6(50 \%)$ \\
\hline Other & $1(25 \%)$ & Surgery & \\
\hline Cycles EURO EWING* & & Yes & $6(50 \%)$ \\
\hline VIDE & $6(3-6)$ & No & $6(50 \%)$ \\
\hline VAI & $5(0-8)$ & Response to surgery $(\mathrm{n}=6)$ & \\
\hline VAC & $6(3-8)$ & Responder & $1(17 \%)$ \\
\hline Cycles full dosage* & $3(1-14)$ & Non-responder & $5(83 \%)$ \\
\hline Cycles full dosage & & Second-line chemotherapy & \\
\hline 1 cycle & $3(25 \%)$ & Irinotecan/Temozolomide & $6(50 \%)$ \\
\hline 2 cycles & $3(25 \%)$ & No therapy & $6(50 \%)$ \\
\hline 3 cycles & $1(8 \%)$ & Current status & \\
\hline 9 cycles & $1(8 \%)$ & Dead & $4(33 \%)$ \\
\hline 10 cycles & $3(25 \%)$ & Alive & $8(67 \%)$ \\
\hline \multirow[t]{2}{*}{14 cycles } & $1(8 \%)$ & Progression-free survival (months) & $19.2(4.1-34.3)$ \\
\hline & & Overall survival (months) & $54.7(21.7-87.6)$ \\
\hline
\end{tabular}

*Data presented as median (range). CR, Complete remission; PR, partial remission; PD, progressive disease; NED, no evidence of disease.

performed every 3 months for the first 3 years, every 6 months up to 5 years and annually thereafter. All patients consented to treatment according to institutional guidelines, as well as to anonymized assessments and analysis of data and outcome of therapy. The study was approved by the Institutional Review Board of the Medical University of Vienna (1971/2019) and complied with good clinical practice guidelines and the Declaration of Helsinki.

Treatment. For patients diagnosed with osteosarcoma, chemotherapy as outlined and published in the randomised phase 3 EURAMOS- 1 trial was used (10). For patients diagnosed with Ewing sarcomas, chemotherapy according to the EURO E.W.I.N.G. protocol was applied (5). Additional supportive therapy for side effect management including granulocyte colony-stimulating factor (G$\mathrm{CSF}$ ) prophylaxis and leukovorin rescue was applied according to clinical requirements and local practice. Cycles were repeated according to the two schedules (EURAMOS-1 and EURO E.W.I.N.G.). Surgical resection of primary tumor was carried out after two cycles of MAP for patients with OSA and after 6 cycles of VIDE for patients with ES. Adjuvant as well as palliative chemotherapy regimens were used according to local practice.

Statistical analysis. Progression-free survival (PFS) was calculated from the date of pre-operative chemotherapy initiation according to the EURAMOS and EURO E.W.I.N.G. protocol until the date of progression, relapse or death, whichever occurred earlier. Overall survival (OS) was calculated from initial diagnosis (date of biopsy) until death. Event-free survival (EFS) was calculated from start of initial diagnosis until progression, relapse or death from any cause. Survival curves were plotted using the Kaplan-Meier method, and survival curves were compared using the log-rank test. Radiologic response for initial metastatic disease was defined using the RECIST criteria (version 1.1): complete response (CR) - no measurable disease; greater than $50 \%$ response - partial response $(\mathrm{PR})$; stable disease $(\mathrm{SD})$ - less than $25 \%$ response or no response and progressive disease (PD) (13).

\section{Results}

Patients with OSA. Detailed patient characteristics are outlined in Table I. Nineteen patients, 11 male (58\%) and 8 female $(42 \%)$, were diagnosed with OSA and the median age was 27 years (range $=18-39$ ). The most frequent tumor site was the lower extremities (58\%). At the time of diagnosis, 16 patients had localized disease $(84 \%)$, whereas 3 patients had primary metastatic disease $(16 \%)$. Out of these three 
Table III. Adverse events.

\begin{tabular}{|c|c|c|c|c|c|c|c|c|}
\hline \multirow{3}{*}{$\begin{array}{l}\text { Characteristic } \\
\text { Toxicity }\end{array}$} & \multicolumn{4}{|c|}{ Osteosarcoma (EURAMOS) } & \multicolumn{4}{|c|}{ Ewingsarcoma (EURO EWING) } \\
\hline & \multicolumn{2}{|c|}{ Grade $1+2$} & \multicolumn{2}{|c|}{ Grade $3+4$} & \multicolumn{2}{|c|}{ Grade $1+2$} & \multicolumn{2}{|c|}{ Grade $3+4$} \\
\hline & $\mathrm{n}$ & $\%$ & $\mathrm{n}$ & $\%$ & $\mathrm{n}$ & $\%$ & $\mathrm{n}$. & $\%$ \\
\hline \multicolumn{9}{|l|}{ Non-haematological toxicity } \\
\hline Nausea & 12 & 63 & 0 & 0 & 8 & 62 & 0 & 0 \\
\hline Fatigue & 11 & 58 & 0 & 0 & 6 & 50 & 0 & 0 \\
\hline Vomiting & 7 & 36 & 0 & 0 & 6 & 50 & 0 & 0 \\
\hline Stomatitis & 8 & 42 & 1 & 5 & 6 & 50 & 0 & 0 \\
\hline Centra/peripheral neurotoxocity & 2 & 10 & 0 & 0 & 0 & 0 & 3 & 25 \\
\hline Elevated kreatinin levels & 3 & 15 & 10 & 52 & 1 & 8 & 1 & 8 \\
\hline Pain & 5 & 26 & 0 & 0 & 3 & 25 & 0 & 0 \\
\hline \multicolumn{9}{|l|}{ Haematological toxicity } \\
\hline Anaemia & 12 & 63 & 1 & 5 & 6 & 50 & 0 & 0 \\
\hline Leukopenia & 9 & 47 & 8 & 42 & 4 & 33 & 0 & 0 \\
\hline Febrile neutropenia & 10 & 52 & 0 & 0 & 4 & 33 & 0 & 0 \\
\hline Thrombocytopenia & 10 & 52 & 0 & 0 & 5 & 41 & 0 & 0 \\
\hline
\end{tabular}

patients, all had lung metastases and 2 patients had additional liver metastases.

All 19 patients were treated according to the EURAMOS protocol as mentioned before. A median of 19 cycles per patient were administered (range $=5-21$ ), but only a median of 9 (range=1-14) cycles at full dose could be given. One patient received only 1 cycle of chemotherapy at full dose, while the maximum number of cycles at full dose was 14 in 1 patient. Thirteen out of 19 patients had a dose reduction to a minimum of $75 \%$, whereas the remaining 6 patients had a dose reduction to $50 \%$. Most frequent reason for dose reductions was elevated methotrexate (MTX) levels due to delayed MTX elimination in 10 of 19 patients. In these patients, the combination of dose reduction of MTX and increased leukovorin rescue led to normalization during the next cycles.

Overall response rate (ORR) in all 19 patients was $90 \%$. Response to chemotherapy in the 3 patients with initially metastatic disease resulted in an ORR of $5 \%$ with 1 patient qualifying for a PR. Tumor response in the 16 out of 19 patients with initially localized disease was CR. One patient with OSA of the maxillary sinus underwent carbon ion therapy after initial chemotherapy resulting in partial remission. Thirteen out of 19 patients underwent surgery after the first 6 cycles of chemotherapy according to the EURAMOS protocol, showing only one responder and 12 (out of 13) non-responders in spite of radiological CR with no evidence of disease on CT and MRI. After relapse of the disease, ifosfamide and pembrolizumab were the most frequently administered second-line therapies at $26 \%$ and $16 \%$, respectively. Response to second-line chemotherapy resulted in $\mathrm{PR}$ in 3 patients, $\mathrm{SD}$ in 5 patients and $\mathrm{PD}$ in the remaining 2 patients requiring further therapy.
Patients with ES. Detailed patient characteristics are outlined in Table II. Twelve patients, 10 male and 2 female, with a median age of 24 years (range $=18-37$ ) were diagnosed with ES. Most frequent tumor sites were the thorax and the pelvis $(n=4$ each,). Eight patients out of 12 had initially localized, whereas 4 patients had initially metastatic disease. All patients in the metastatic setting presented with lung metastases. Patients received a median of 6 cycles of VIDE (range=3-6), male patients a median of 5 cycles VAI (range $=0-8$ ) and female patients a median of 8 cycles VAC (range=3-8).

A median of 3 chemotherapy cycles were given at full dose (range $=1-14)$. No dose reduction was necessary in only 3 out of the 12 patients; one patient received all 14 cycles at $100 \%$, whereas the remaining 2 patients stopped chemotherapy after 9 and 10 cycles, due to progression without dose reduction. The remaining 9 patients required dose reductions to $75 \%(n=7)$ and to $50 \%(\mathrm{n}=2)$. The most frequent reason for dose reduction was prolonged aplasia ( 6 out of 12 patients). All 3 patients with ifosfamide-induced neurotoxicity were successfully treated with intravenous methylene blue. Response to chemotherapy for patients with an initially metastatic setting resulted in an ORR of $33 \%$, with one patient achieving a CR. One patient with localized disease had progressive disease after VIDE. Six out of 12 patients underwent surgery after neoadjuvant VIDE. One patient was rated as a histological responder, whereas the remaining 5 out of 6 patients were non-responders.

Toxicity. Severe toxicities were rare in both protocols, as mostly grade 1 and 2 toxicities were encountered. The most common grade 3 and 4 toxicities in patients with OSA were renal, i.e. elevated creatinin levels (52\%) due to protracted MTX elimination. The most common grade 3 and 4 hematologic toxicities were leukopenia (42\%) and anemia (5\%). By contrast, 


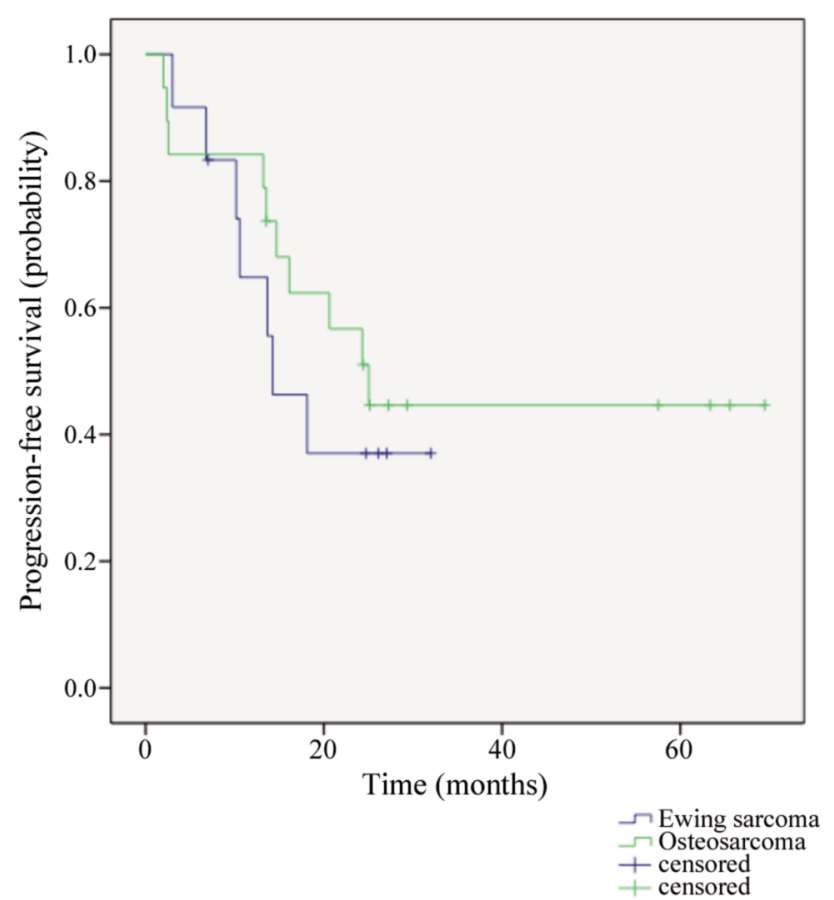

Figure 1. Progression-free survival (PFS) in osteosarcoma (OSA) and Ewing sarcoma (ES). Median PFS for patients with OSA was not reached and median PFS for ES was 19.2 months (range=4.1-34.3).

the most common grade 3 and 4 non-hematologic toxicity in patients with ES was central/peripheral neurotoxicity due to high dose ifosfamide (25\%). The most commonly encountered hematologic adverse events were grade 1 and 2 toxicities, namely anemia (50\%) followed by thrombocytopenia (41\%) (Table III).

Survival. Median PFS (Figure 1) and OS (Figure 2) in patients with OSA were not reached (n.r.) and 36.3 months (range=11.7-60.9). Median PFS and OS for patients with ES were 19.2 months (range=4.1-34.3) (Figure 1) and 54.7 months (range $=21.7-87.6$ ) (Figure 2), respectively.

\section{Discussion}

The objective of this retrospective analysis was to evaluate the efficacy and safety of EURO E.W.I.N.G and EURAMOS-1 in AYA patients, in a real world setting, as these protocols have shown promising efficacy in patients with ES and OSA, respectively $(5,9,10)$.

In the past, various clinical trials have assessed the activity and safety of these protocols that are routinely applied at our institution. In the EURO-EWING99-R1 trial, cyclophosphamide was compared to ifosfamide as consolidation after induction therapy with VIDE (14). In that

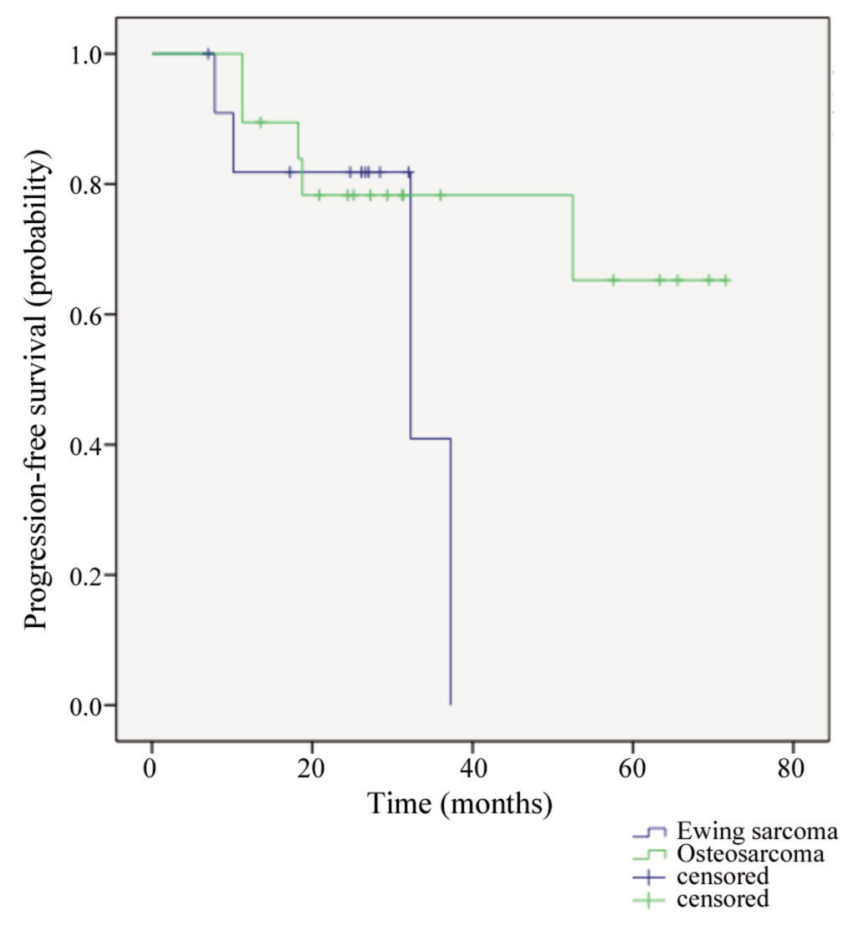

Figure 2. Overall survival (OS) in osteosarcoma (OSA) and Ewing sarcoma (ES). Median OS for patients with OSA was 36.3 months (range=11.7-60.9 months) and median OS for patients with ES was 54.7 months (range $=21.7-87.6$ ).

trial, VAI and VAC were not inferior but VAC was slightly worse than VAI in men and vice versa. In addition, $4 \%$ of patients experienced a major switch between treatment arms. The main reasons were renal toxicity followed by patients' choice and other toxicities, while neurological symptoms were only rarely encountered (14). In the present study, a higher percentage of dose reductions to $75 \%$ was observed.

Juergens et al. investigated the safety of the EUROE.W.I.N.G. protocol. In this trial, 851 patients aged $\leq 49$ years were randomized (15). In this trial, a switch from VAI to VAC was necessary in 30 patients and from VAC to VAI in 3 patients. The most common reason for switching from VAI to VAC was renal toxicity due to ifosafmide. Central and peripheral neurotoxicity grade 3 and 4 were rare in this trial ( $0.5 \%$ and $0.4 \%$, respectively). When neurotoxicity occurred, ifosfamide was stopped and replaced with cyclophosphamide. Neurotoxicity in our study was managed with dose reduction and additional therapy with methylen-blue, resulting in resolution of symptoms without the necessity to replace ifosfamide by the less neurotoxic cyclophosphamide.

Pieper et al. compared 47 patients with ES aged $>40$ years with adolescents and demonstrated a similar outcome with comparable side effects (16). In their retrospective study, patients received either EICCESS 92 or EURO-E.W.I.N.G. 99. Thirty-one patients received EURO-E.W.I.N.G. 99. The 
most common grade 3 and 4 hematologic toxicity was neutropenia for VIDE as well for VAI/VAC. Treatment delays (more than 5 days postponement) were only reported for patients receiving VIDE. Dose reductions were not described in this study (16). In contrast to these findings, hematologic toxicities greater than grade 2 were not documented in our patient cohort. A potential explanation might be the fact that dose reductions were performed in case of side effects. No treatment delays were necessary in our patients.

For patients with ES, the most frequent grade 1 and 2 hematologic toxicities were anemia and thrombocytopenia, which was in accordance with study conducted by Juergens et al. (15). The most frequent grade 1 and 2 toxicities reported by Juergens et al. were anemia and thrombocytopenia, while the most frequent non-hematologic grade 3 and 4 toxicities were stomatitis and vomiting (15).

For patients with OSA, the EURAMOS-1 schedule is a standard of care consisting of preoperative chemotherapy followed by surgical resection and postoperative chemotherapy as stated in the Patients and Methods section. The EURAMOS1 protocol has been assessed in two, open label, phase III trials $(10,17)$.

Whelan et al. investigated preoperative MAP followed by postoperative MAP in combination with ifosfamide and etoposide (MAPIE) versus postoperative MAP versus MAP in combination with pegylated interferon. In this phase III trial, 1334 out of 2260 patients randomized were younger than 40 years. The most common grade 3 and 4 side effects due to preoperative chemotherapy consisted of neutropenia and infection (17).

Marina et al. investigated the effect of MAPIE versus MAP in the postoperative setting in the same population (10). In summary, a dose intensive postoperative chemotherapy with MAPIE did not improve event-free survival. Moreover, patients had more toxicities in the MAPIE arm. Thus, patients are currently given only MAP in the pre- as well in the postoperative setting according to these results. The most common grade 4 hematologic toxicities in the MAP arm were neutropenia followed by thrombocytopenia. In this trial, $75 \%$ of patients received methotrexate, $83 \%$ doxorubicin and $92 \%$ cisplatin at the planned target number of doses in the adjuvant setting (10). In this analysis, treatment delays were necessary in $58 \%$ of patients. Central neuropathy attributable to ifosfamide was comparable to our series (13\% versus $10 \%$ ).

In the present study, the most common grade $3 / 4$ hematologic toxicity in patients with OSA was leukopenia (42\%), which was less than the study by Marina et al. (82\%). A reason for the lower incidence might have been the dose reductions due to hematologic toxicities in our patients (10). Stomatitis as side effect was similar in our study when compared to the study by Marina (42\% versus $44 \%$ ). Grade 1 and 2 as well as grade 3 and 4 elevated creatinine levels occurred in $15 \%$ and $53 \%$ of our patients, respectively. Grade
1 and 2 toxicites were similar when compared to Marina et al. In our patients more grade 3 and 4 renal toxicities could be observed, which were nevertheless short and manageable with hydration and higher leukovorin substitution.

Raze et al. investigated the role of age in cancer survival. Different tumors in AYAs were investigated between 2000 and 2016 in France. For AYA patients with ES and OSA, the 5 -year OS was around $60 \%$ and lower (18). White et al. underlined that AYA patients with rare sarcomas, including ES and OSA should be treated in reference centers (19).

The most important limitations of our study are its retrospective nature as well as the small patient population when compared to multicentric prospective phase III trials. Further larger prospective studies should be performed to evaluate risk factors like age, gender, and laboratory parameters in this patient population.

In summary, both protocols, EURO E.W.I.N.G. and EURAMOS-1, were well tolerated in a real life-setting. However, dose reductions should be considered instead of dose delays when side effects, such as delayed MTX elimination, prolonged aplasia or neurotoxicty due to ifosfamide, are noticed. Nevertheless, since side effects are unpredictable and specialized management is necessary, patients with rare bone sarcomas should be treated in reference centers.

\section{Conflicts of Interest}

The Authors declare no conflicts of interest.

\section{Authors' Contributions}

Study concept and design were performed by Christoph Minichsdorfer and Wolfgang Lamm. Oskar Steinbrecher carried out acquisition of data. Analysis and interpretation of data were conducted by all authors. Drafting of the manuscript was performed by Christoph Minichsdorfer, Oskar Steinbrecher, and Wolfgang Lamm. Critical revision of the manuscript was carried out by all Authors.

\section{References}

1 Ewing J: The Classic: Diffuse endothelioma of bone. Proceedings of the New York Pathological Society 1921;12:17. Clin Orthop Relat Res 450: 25-27, 2006. PMID: 16951641. DOI: 10.1097/01.blo.0000229311.36007.c7

2 Cangir A, Vietti TJ, Gehan EA, Burgert EO, Jr., Thomas P, Tefft M, Nesbit ME, Kissane J and Pritchard D: Ewing's sarcoma metastatic at diagnosis. Results and comparisons of two intergroup Ewing's sarcoma studies. Cancer 66: 887-893, 1990. PMID: 2201433. DOI: 10.1002/1097-0142(19900901)66:5 $<887$ ::aid-cncr2820660513>3.0.co;2-r

3 Rosen G, Wollner N, Tan C, Wu SJ, Hajdu SI, Cham W, D’Angio GJ and Murphy ML: Proceedings: Disease-free survival in children with Ewing's sarcoma treated with radiation therapy and adjuvant four-drug sequential chemotherapy. Cancer 33: 384-393, 1974. PMID: 4812758. DOI: 10.1002/10970142(197402)33:2<384::aid-cncr2820330213>3.0.co;2-t 
4 Ganjoo KN and Patel S: The treatment outcome for adult patients with Ewing's sarcoma. Curr oncol rep 15: 372-377, 2013. PMID: 23605781. DOI: 10.1007/s11912-013-0317-5

5 Whelan J, Le Deley MC, Dirksen U, Le Teuff G, Brennan B, Gaspar N, Hawkins DS, Amler S, Bauer S, Bielack S, Blay JY, Burdach S, Castex MP, Dilloo D, Eggert A, Gelderblom H, Gentet JC, Hartmann W, Hassenpflug WA, Hjorth L, Jimenez M, Klingebiel T, Kontny U, Kruseova J, Ladenstein R, Laurence V, Lervat C, Marec-Berard P, Marreaud S, Michon J, Morland B, Paulussen M, Ranft A, Reichardt P, van den Berg H, Wheatley K, Judson I, Lewis I, Craft A, Juergens H and Oberlin O: Highdose chemotherapy and blood autologous stem-cell rescue compared with standard chemotherapy in localized high-risk Ewing sarcoma: Results of Euro-E.W.I.N.G.99 and Ewing-2008. J Clin Oncol 36(31): 3110-3119, 2018. PMID: 30188789. DOI: 10.1200/JCO.2018.78.2516

6 Mirabello L, Troisi RJ and Savage SA: International osteosarcoma incidence patterns in children and adolescents, middle ages and elderly persons. Int $\mathrm{J}$ cancer 125: 229-234, 2009. PMID: 19330840 . DOI: $10.1002 / \mathrm{ijc} .2430$

7 Kager L, Zoubek A, Potschger U, Kastner U, Flege S, KempfBielack B, Branscheid D, Kotz R, Salzer-Kuntschik M, Winkelmann W, Jundt G, Kabisch H, Reichardt P, Jurgens H, Gadner H, Bielack SS and Cooperative German-Austrian-Swiss Osteosarcoma Study G: Primary metastatic osteosarcoma: presentation and outcome of patients treated on neoadjuvant Cooperative Osteosarcoma Study Group protocols. J Clin Oncol 21: 2011-2018, 2003. PMID: 12743156. DOI: $10.1200 / \mathrm{JCO} .2003 .08 .132$

8 Cho WH, Song WS, Jeon DG, Kong CB, Kim MS, Lee JA, Yoo JY, Kim JD and Lee SY: Differential presentations, clinical courses, and survivals of osteosarcomas of the proximal humerus over other extremity locations. Ann Surg Oncol 17: 702-708, 2010. PMID: 19921336. DOI: 10.1245/s10434-009-0825-6

9 Smeland S, Bruland OS, Hjorth L, Brosjo O, Bjerkehagen B, Osterlundh G, Jakobson A, Hall KS, Monge OR, Bjork O and Alvegaard TA: Results of the Scandinavian Sarcoma Group XIV protocol for classical osteosarcoma: 63 patients with a minimum follow-up of 4 years. Acta Orthopaedica 82: 211-216, 2011. PMID: 21434784. DOI: 10.3109/17453674.2011.566141

10 Marina NM, Smeland S, Bielack SS, Bernstein M, Jovic G, Krailo MD, Hook JM, Arndt C, van den Berg H, Brennan B, Brichard B, Brown KLB, Butterfass-Bahloul T, Calaminus G, Daldrup-Link HE, Eriksson M, Gebhardt MC, Gelderblom H, Gerss J, Goldsby R, Goorin A, Gorlick R, Grier HE, Hale JP, Hall KS, Hardes J, Hawkins DS, Helmke K, Hogendoorn PCW, Isakoff MS, Janeway KA, Jurgens H, Kager L, Kuhne T, Lau CC, Leavey PJ, Lessnick SL, Mascarenhas L, Meyers PA, Mottl H, Nathrath M, Papai Z, Randall RL, Reichardt P, Renard M, Safwat AA, Schwartz CL, Stevens MCG, Strauss SJ, Teot L, Werner M, Sydes MR and Whelan JS: Comparison of MAPIE versus MAP in patients with a poor response to preoperative chemotherapy for newly diagnosed high-grade osteosarcoma (EURAMOS-1): an open-label, international, randomised controlled trial. The Lancet 17: 1396-1408, 2016. PMID: 27569442. DOI: 10.1016/S1470-2045(16)30214-5

11 Gasparini M: High-dose ifosfamide alone and in combination for solid malignancies in childhood. Cancer Chemother Pharmacol 18(Suppl 2): S18, 1986. PMID: 3102090. DOI: 10.1007/BF006 47442

12 Goorin AM, Harris MB, Bernstein M, Ferguson W, Devidas M, Siegal GP, Gebhardt MC, Schwartz CL, Link M and Grier HE:
Phase II/III trial of etoposide and high-dose ifosfamide in newly diagnosed metastatic osteosarcoma: a pediatric oncology group trial. J Clin Oncol 20: 426-433, 2002. PMID: 11786570. DOI: 10.1200/JCO.2002.20.2.426

13 Eisenhauer EA, Therasse P, Bogaerts J, Schwartz LH, Sargent D, Ford R, Dancey J, Arbuck S, Gwyther S, Mooney M, Rubinstein L, Shankar L, Dodd L, Kaplan R, Lacombe D and Verweij J: New response evaluation criteria in solid tumours: revised RECIST guideline (version 1.1). Eur J Cancer 45: 228247, 2009. PMID: 19097774. DOI: 10.1016/j.ejca.2008.10.026

14 Le Deley MC, Paulussen M, Lewis I, Brennan B, Ranft A, Whelan J, Le Teuff G, Michon J, Ladenstein R, Marec-Berard $\mathrm{P}$, van den Berg H, Hjorth L, Wheatley K, Judson I, Juergens H, Craft A, Oberlin O and Dirksen U: Cyclophosphamide compared with ifosfamide in consolidation treatment of standard-risk Ewing sarcoma: results of the randomized noninferiority EuroEWING99-R1 trial. J Clin Oncol 32: 2440-2448, 2014. PMID: 249824645. DOI: 10.1200/JCO.2013.54.4833

15 Juergens C, Weston C, Lewis I, Whelan J, Paulussen M, Oberlin O, Michon J, Zoubek A, Juergens $\mathrm{H}$ and Craft A: Safety assessment of intensive induction with vincristine, ifosfamide, doxorubicin, and etoposide (VIDE) in the treatment of Ewing tumors in the EURO-E.W.I.N.G. 99 clinical trial. Pediatr Blood Cancer 47: 22-29, 2006. PMID: 16572519. DOI: 10.1002/pbc.2080

16 Pieper S, Ranft A, Braun-Munzinger G, Jurgens H, Paulussen M and Dirksen U: Ewing's tumors over the age of 40: a retrospective analysis of 47 patients treated according to the International Clinical Trials EICESS 92 and EURO-E.W.I.N.G. 99. Onkologie 31: 657-663, 2008. PMID: 19060503. DOI: $10.1159 / 000165361$

17 Whelan JS, Bielack SS, Marina N, Smeland S, Jovic G, Hook JM, Krailo M, Anninga J, Butterfass-Bahloul T, Bohling T, Calaminus G, Capra M, Deffenbaugh C, Dhooge C, Eriksson M, Flanagan AM, Gelderblom H, Goorin A, Gorlick R, Gosheger G, Grimer RJ, Hall KS, Helmke K, Hogendoorn PC, Jundt G, Kager L, Kuehne T, Lau CC, Letson GD, Meyer J, Meyers PA, Morris C, Mottl H, Nadel H, Nagarajan R, Randall RL, Schomberg P, Schwarz R, Teot LA, Sydes MR, Bernstein M and collaborators: EURAMOS-1, an international randomised study for osteosarcoma: results from pre-randomisation treatment. Ann Oncol 26: 407-414, 2015. PMID: 25421877. DOI: 10.1093/ annonc/mdu526

18 Raze T, Lacour B, Cowppli-Bony A, Delafosse P, Velten M, Trétarre B, Defossez G, Hammas K, Woronoff AS, Ganry O, Plouvier S, Coureau G, Guizard AV, Bara S, Monnereau A, Daubisse-Marliac L, Troussard X, D’Almeida T, Baldi I, Bouvier V, Boissel N, Clavel J and Desandes E: Cancer among adolescents and young adults between 2000 and 2016 in France: Incidence and improved survival. J Adolesc Young Adult Oncol, 2020. PMID: 32412825. DOI: 10.1089/jayao.2020.0017

19 White VM, Orme LM, Skaczkowski G, Pinkerton R, Coory M, Osborn M, Bibby H, Nicholls W, Conyers R, Phillips MB, Harrup R, Walker R, Thompson K and Anazodo A: Management of sarcoma in adolescents and young adults: An Australian population-based study. J Adolesc Young Adult Oncol 8(3): 272280, 2019. PMID: 30822262. DOI: 10.1089/jayao.2018.0136

Received July 26, 2020

Revised October 5, 2020

Accepted October 23, 2020 